\title{
The morphology of choroidal neovascularization in chronic central serous chorioretinopathy presenting with flat, irregular pigment epithelium detachment
}

\author{
Claudio Azzolini · Jennifer Cattaneo - Laura Premoli • Cristian Metrangolo • \\ Maurizio Chiaravalli $\cdot$ Simone Donati $[$ )
}

Received: 10 June 2020/ Accepted: 5 March 2021/Published online: 21 March 2021

(C) The Author(s) 2021

\begin{abstract}
Purpose To evaluate morphological characteristics of choroidal neovascularization in chronic central serous chorioretinopathy (CSC) presenting with flat and irregular pigment epithelium detachment (FIPED) by means of innovative multimodal imaging.

Methods In this observational cross-sectional study, we examined 10 consecutive patients affected by chronic CSC and FIPED using fluorescein angiography (FA), indocyanine-green angiography (ICGA) and optical coherence tomography angiography (OCTA). A qualitative analysis of the nature and characteristics of neovascular membrane was performed, combining available multimodal imaging and literature data.

Results Multiple areas of retinal pigment epithelium alterations, macular hypo- and hyperpigmentation and atrophic areas were identified. Spectral domain OCT (SD-OCT) showed subretinal fluid in $80 \%$ of eyes and the 'double layer sign' in all patients. Late FA phases showed staining areas without leakage in all eyes; ICGA showed a hyperfluorescent plaque
\end{abstract}

Presented as a paper at the 77th Macula Society Meeting, Los Angeles CA, USA, 21st--24th February 2018.

C. Azzolini · J. Cattaneo $\cdot$ L. Premoli .

C. Metrangolo $\cdot$ M. Chiaravalli $\cdot$ S. Donati $(\bowtie)$

Ophthalmology Clinic, Department of Medicine and

Surgery, University of Insubria - ASST Sette Laghi,

Varese, Italy

e-mail: simone.donati@unisubria.it with surrounding hypofluorescence in $80 \%$ of patients. OCTA detected characteristic neovascular networks in the outer retina within the FIPEDs, classified as filamentous vessels with a pruned tree-like pattern in five eyes and a tangled pattern in three eyes. The choriocapillaris network showed dark areas in $80 \%$ of eyes and diffuse dark spots in all eyes.

Conclusion Multimodal imaging completes clinical characterization of FIPEDs in chronic CSC. This study using OCTA technology describes the phenotype of hidden neovascular lesions in shape and morphology.

Keywords Central serous chorioretinopathy Choroidal neovascularization - OCT angiography · Pigment epithelium detachment

\section{Introduction}

Central serous chorioretinopathy (CSC) is a posterior segment disease characterized by serous detachments of the neurosensory retina $[1,2]$. CSC has been classified into two main types: acute CSC and chronic CSC. In acute CSC, self-resolving subretinal detachment is observed within four months from the onset of symptoms [3]. In chronic CSC, we know that widespread retinal pigment epithelium (RPE) alterations are present, which may or may not be associated with serous retinal detachment. Several, deep changes in 
the RPE structure are diagnosed with the presence of areas of atrophy and pigment epithelium detachments (PEDs). Moreover, chronic CSC can be complicated by choroidal neovascularization (CNV) $[4,5]$. A multimodal diagnostic approach represents in these cases an interesting example of clinical evaluation, in particular when chronic CSC is complicated by flat and irregular RPE detachments and neovascularization [6, 7]. Fluorescein angiography (FA) and indocyanine-green angiography (ICGA), together with structural optical coherence tomography (OCT), are not always able to explain subretinal fluid persistence and PED elevation [8, 9]. The application of OCT angiography (OCTA) could give more clinical and prognostic data to assess the presence of CNV in CSC, but there are variable and non-comparable results [10]. Different authors have described a variable prevalence of CNV, combining FA and OCTA. Difficulties come from the diffuse leakage of RPE, the slight RPE detachment and ICGA detection. Quaranta et al. identified CNV applying OCTA in the full sample of seven chronic CSC eyes with 'slight retinal PED with small undulations' [11] and Dansingani et al. in 95\% of 22 eyes with pachychoroid diseases and 'shallow irregular pigment epithelial detachments' [12]. Moreover, CNVs may show different behaviors and clinical morphologies due to chronic subretinal fluid, flat, irregular pigment epithelium detachment (FIPED) and stability during follow-up [13].

Few data are available in literature investigating $\mathrm{CNV}$ morphology and characteristics in CSC, in particular in cases with slight PED [14-16].

The aim of this study is to investigate and recognize clinical characteristics of choroidal new vessels in FIPED complicating chronic CSC, using multimodal imaging.

\section{Materials and methods}

This observational cross-sectional study enrolled a cohort of 10 consecutive patients with a diagnosis of chronic CSC presenting with an irregular PED, examined at our Medical Retinal Service, Ophthalmology Clinic, University of Insubria, ASST Sette Laghi, Varese, Italy. Inclusion criteria were the diagnosis of chronic CSC with the presence of FIPEDs. We defined chronic CSC as the presence of diffuse defects of RPE, whether or not they were associated with a variable persistence of subretinal fluid involving the foveal area, for more than six months. ICGA examination may show in these cases typical extramacular hypo- and hyperfluorescent areas. Exclusion criteria were signs of age-related macular degeneration (AMD), like macular drusen, atrophic macular areas, presence of intra- or subretinal hemorrhages, also considering the fellow eye. We excluded all patients showing other associated, previous or concomitant ophthalmological conditions that could influence clinical and imaging analysis, such as retinal vascular pathologies, vitreoretinal interface diseases and presence of significant media opacity, as well as non-collaborative patients.

All patients were enrolled in our study after a visit in our Medical Retina Service. The patients were not followed directly; rather, they came for a second opinion consultation or for more in-depth ophthalmological examinations from external colleagues. All patients reported in their clinical history a diagnosis of acute CSC at least six months old. Previous clinical data, including FA and ICGA, showed clinical signs of acute self-limited CSC. The patients were treatmentnaïve apart from medical therapy, such as diuretics or nutritional supplementation. None of the enrolled patients had been previously evaluated with OCTA.

Written informed consent was obtained from all patients in compliance with the tenets of the Declaration of Helsinki. The institutional review board approved all study-related data acquisition.

After enrolment, each patient underwent a complete ophthalmological examination, including Snellen Best-Corrected Visual Acuity (BCVA) evaluation. At the same visit, we performed FA, ICGA, SD-OCT and OCTA examinations. Structural SD-OCT was performed with the Zeiss Cirrus OCT device in both eyes of each patient using the following protocol: macular map and $6 \mathrm{~mm}$ horizontal and vertical scan lines centered on the fovea. On OCT structural imaging, we defined FIPED as an irregular elevation of the RPE allowing visualization of the Bruch's membrane. Angiography examinations were performed using HRA2 (Heidelberg, Germany) and completed with blue light autofluorescence and redfree images. FA and ICGA were performed on all patients. ICG was injected at a dose of $2.5 \mathrm{mg}$. Imaging was captured at early (5 min), medium (15 min) and late phases $(25 \mathrm{~min})$. The identification and classification of $\mathrm{CNV}$ were performed according 
to the literature. OCTA was performed using the AngioVue OCTA system on the commercially available RTVue XR Avanti device (Optovue, Inc, Fremont, CA), and images were analyzed using the latest version of the software. This instrument has an A-scan rate of 70,000 scans per second to acquire OCTA volumes consisting of 30,304 A-scans in approximately $2.6 \mathrm{~s}$. Orthogonal recording and merging of two consecutive scan volumes were used to obtain $3 \times 3 \mathrm{~mm}$ and $6 \times 6 \mathrm{~mm}$ OCTA volumes centered on the fovea. OCT angiograms were co-recorded with the corresponding OCT B-scans. Each raw retinal scan set was subsequently evaluated by two masked expert ophthalmologists (JC, SD) to assess the presence of vascular alterations or neovascular proliferations on each layer. Type and characteristics of neovascular lesion were described and compared by both clinicians.

We used descriptive statistics for demographic and clinical data (Microsoft Excel for Mac, version 14.0, 2011, Redmond, USA). Student's t-test was applied for comparison and the Pearson test for correlation. A $p$ value of $<0.05$ was considered for statistical significance.

\section{Results}

Demographics are listed in Table 1. Ten eyes of 10 patients were diagnosed with chronic CSC presenting with a FIPED. Mean BCVA was 20/36 $(0.26 \pm 0.15$ LogMAR). Mean follow-up time from diagnosis to enrolment date was $8.3 \pm 2.1$ months.

Fundus examination revealed the presence of subretinal fluid involving the macular region in $80 \%$ of eyes; all eyes presented multiple zones of RPE alterations, in particular macular hypo- and hyperpigmentation and atrophic areas. Neither drusen nor subretinal hemorrhages were detected in any patient.

SD-OCT B-scans clearly showed subretinal fluid in $80 \%$ of eyes. Two patients did not present with fluid at the time of enrolment. FIPEDs were diagnosed, presenting as the typical double layer sign in all patients. Mean central retinal thickness was $280.3 \pm 58.47 \mu \mathrm{m}$, and mean foveal choroidal thickness was $368 \pm 16.81 \mu \mathrm{m}$ (Fig. 1A).

Fundus autofluorescence showed a granular hypoand hyperfluorescent pattern in the macular region in all patients (Fig. 1B).

FA revealed alternating hyperfluorescent and hypofluorescent spots in the early phases; late phase angiograms showed staining areas without leakage in all eyes (Fig. 1C, D).

ICGA found a hyperfluorescent plaque with surrounding areas of hypofluorescence in $80 \%$ of eyes. No signs of leakage or choroidal polyps were evident on mid or late stage angiograms (Fig. 1E, F).

OCTA revealed normal retinal circulation and normal features of both the superficial and deep capillary plexus in all of the eyes examined. The

Table 1 Demographics and clinical data of enrolled patients

\begin{tabular}{llllllll}
\hline Patient & Age & Sex & Visual acuity & CT $(\mu \mathrm{m})$ & CRT $(\mu \mathrm{m})$ & Presence of SRF & Type of PED \\
\hline 1 & 68 & $M$ & $20 / 40$ & 384 & 296 & Yes & Irregular \\
2 & 58 & $M$ & $20 / 30$ & 385 & 276 & Yes & Irregular \\
3 & 67 & $M$ & $20 / 30$ & 380 & 228 & No & Irregular \\
4 & 74 & $M$ & $20 / 30$ & 378 & 234 & Yes & Irregular \\
5 & 76 & $F$ & $20 / 50$ & 340 & 334 & Yes & Irregular \\
6 & 68 & $M$ & $20 / 30$ & 357 & 294 & No & Irregular \\
7 & 64 & $M$ & $20 / 25$ & 350 & 252 & Yes & Irregular \\
8 & 76 & $F$ & $20 / 63$ & 354 & 307 & Yes & Irregular \\
9 & 73 & $F$ & $20 / 30$ & 366 & 189 & Yes & Irregular \\
10 & 73 & $F$ & $20 / 63$ & 386 & 393 & Yes & Irregular \\
Mean & $69,7 \pm 5,79$ & & $20 / 36$ & $368 \pm 16,81$ & $280,3 \pm 58,47$ & & \\
values & & & $(0,26 \pm 0,15 L \lg$ MAR) & & & & \\
\hline
\end{tabular}

$C T$ choroidal thickness, $C R T$ central retinal thickness, $S R F$ subretinal fluid, $P E D$ pigment epithelium detachment 
algorithm segmentation of the outer retina and choriocapillaris showed signal and flow alterations in all eyes: in particular, we observed a distinct neovascular network that we classified as filamentous vessels in $80 \%$ of the eyes (Fig. 1G-L). Considering the morphology of these networks, we identified a pruned tree-like pattern in five eyes, while in three eyes the morphology resembled a tangled pattern with a high flow and a 'ball of wool' shape (Fig. 2A, B). All the identified networks were detected in the outer retina segmentation layer, in correspondence with the FIPED. Based on the analysis of the choriocapillaris vascular network, we identified the presence of more extended OCTA signal alterations: dark areas in $80 \%$ of the eyes and diffuse dark spots in all eyes.

\section{Discussion}

Our study collected a consecutive series of patients with flat, irregular PEDs in chronic CSC. This morphology is likely to be observed in chronic CSC and differs from the dome shaped PEDs typical of the acute form: it has been variously defined as irregular wave, rough or dimpled surface. It could also present in AMD, together with other typical signs of $\mathrm{CNV}$ [13].

In our patients, we observed widespread alterations involving the retina and choriocapillary complex. These pathological features have been already detected by FA and ICGA, but the structural OCT and OCTA examinations provide a more complete description. This approach represents an example of multimodal imaging, where each examination could reveal different clinical and morphological features.

FA showed multiple staining points corresponding to nonspecific areas of hyperpermeability. $80 \%$ of eyes a hyperfluorescent plaque with surrounding areas of hypofluorescence was observed in the late phase of ICGA angiography; 20\% of eyes exhibited an ICG hypofluorescent washout. However, the persistence or vanishing of choroidal staining is difficult to evaluate in the late stage angiograms in presence of RPE atrophy, as previously described [16]. No signs of leakage or choroidal polyps were evident on the mid or late stage angiograms. In his retrospective multiimaging study, Hage examined 53 cases of chronic CSC presenting with FIPEDs in an attempt to discriminate avascular flat, irregular PEDs from those in which the PEDs were consistent with type $1 \mathrm{CNV}$ [6]. ICG evaluation only found $\mathrm{CNV}$ in ten eyes, with all the others considered avascular flat PEDs. Structural OCT revealed different retinal pathological characteristics in our patients: the presence of subretinal fluid in $80 \%$ of eyes and PED with a flat and undulated structure $[17,18]$.

Gupta et al. [15], following the studies by Bousquet [19], tried to correlate the reflectivity pattern between the undulating RPE and underlying Bruch's membrane with the presence of neovascular tissue complex. The presence of a clear hyperreflective RPE layer with a hyporeflective middle layer and a slightly thickened Bruch's membrane, the so called 'double layer sign,' suggested the possibility of neovascular complications. This sign has been previously described as a pathological feature in polypoidal choroidal vasculopathy and PEDs in pachychoroid diseases [14], representing the neovascular proliferation and exudation from the polyps [20]. Chug et al. discussed the convergent mechanism of formation between CSC and polyps due to hyalinization of choroidal vessels, along with replacement of the smooth muscle component by amorphous pseudocollagenous tissue [14-21].

Today, OCTA is able to complete the multimodal approach, especially in chronic CSC and pachychoroid diseases, where CNV is difficult to be detected by FA or ICGA [16-23]. In our study, the analysis with segmentation of the outer retina revealed the presence of pathological vascular networks in $80 \%$ of eyes in correspondence with the flat, irregular PEDs. Our observation is in line with the findings reported by Quaranta et al., who used OCTA to analyze 12 eyes, seven with flat, irregular PEDs and five without PEDs. Their analysis revealed neovascular patterns in all seven eyes with PEDs [11]. Recent work by Tadayoni and Liu underlined the added value of OCTA over standard FA and ICGA, in detecting the presence of $\mathrm{CNV}$ in FIPED more frequently and with greater sensitivity [24, 25]. Our study is the first in the literature to apply OCTA to describe the shape and structure of these abnormal vascular networks in patients affected by CSC presenting with FIPEDs. We found two different morphologies: filamentous vessels with a main neovascular trunk and a few branches that we defined as a pruned tree-like type (62.5\% of cases), and filamentous vessels with a thick net and wool-like appearance that we defined as a 'tangled pattern' 


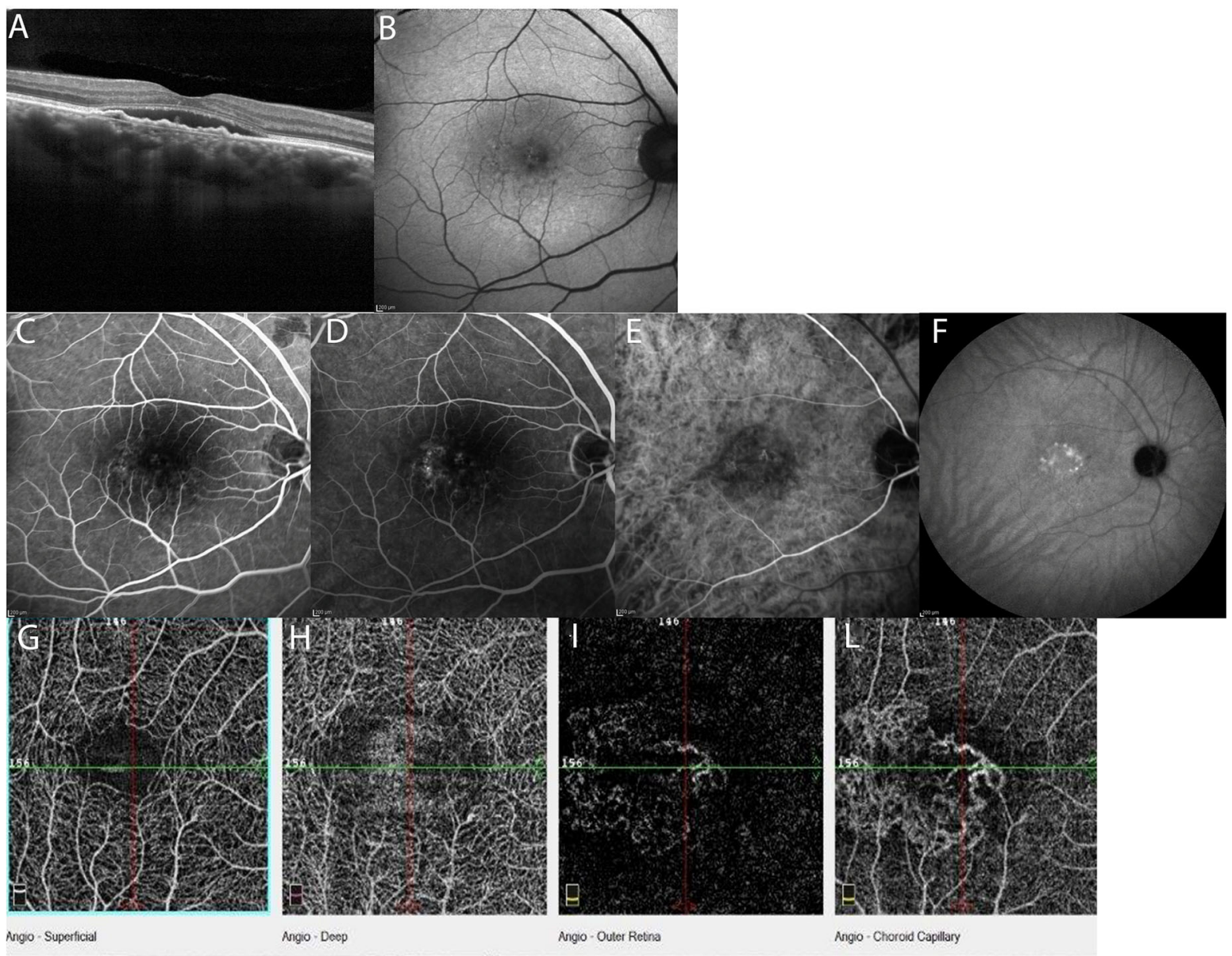

Fig. 1 Multimodal imaging of a flat, irregular retinal epithelium detachment secondary to chronic serous chorioretinopathy (Patient $n^{\circ}$ 6). A- OCT B scan showing subretinal fluid and flat

$(37.5 \%$ ) (Fig. 2A, B). Miere and Souied, in 2016, describing the OCTA features of vascular structure in fibrotic AMD lesions, identified similar networks [24, 26]. Karacorlu, in 2019, described the same pattern of filamentous vessels in fibrotic and low activity neovascular lesions in AMD [27]. Correct recognition of neovascular patterns may be useful to distinguish type $1 \mathrm{CNV}$ of low grade activity to type 1 $\mathrm{CNV}$ at early stages where the progression with subretinal and sub-RPE fluid may be more aggressive or responsive to photodynamic therapy or anti-VEGF [14-16].

The liaison with the morphology of the new vessels recognized in fibrotic scars of AMD lesions is natural: similar proliferative stimuli are present in chronic pathologies, such as choriocapillaris degeneration and and irregular PED; B Fundus autofluorescence; C, D FA at different stages; E, F ICGA at early and late phases; G-L OCTA images showing retinal vasculature patterns in different slabs

vascular hypoperfusion, which can lead to vascular proliferation. A very important finding has been the identification of such proliferation in a significant percentage of chronic CSC patients showing PEDs, to explain the poor prognosis of these cases. A long duration of PED could lead to the disruption of Bruch's membrane and subsequently promote the formation of $\mathrm{CNV}[13,14]$. Our theory is also supported by the studies by Miere and Costanzo describing the OCTA features of subretinal fibrosis in AMD and neovascular complications in CSC [24, 28].

In this study, we also described two different characteristics of the choriocapillaris in OCTA, dark areas and dark spots (Fig. 3A, B). We believe that these aspects may be typical of chronic CSC, in which the RPE degeneration and choriocapillary alterations 


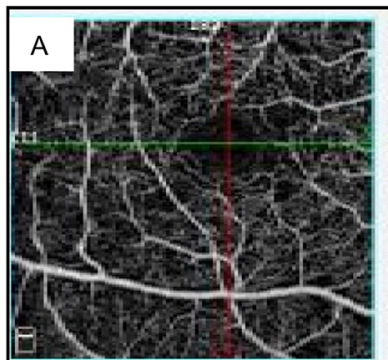

ngo- Expeticial

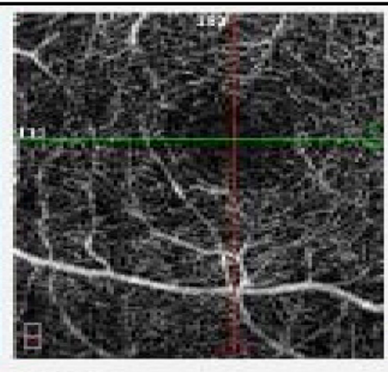

Maga-Desep

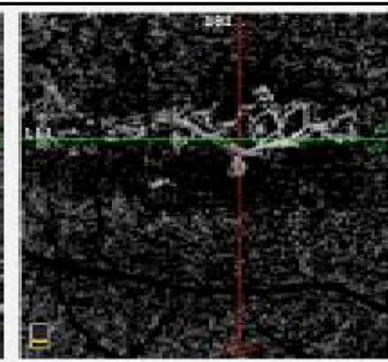

thgo - Duter fivering

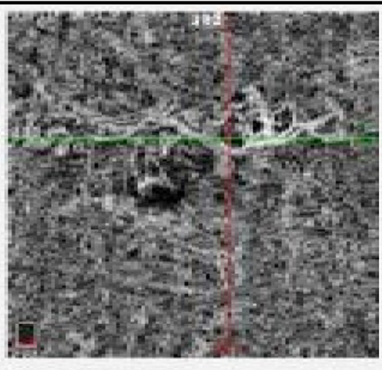

Anga-Chorsel Capiliary

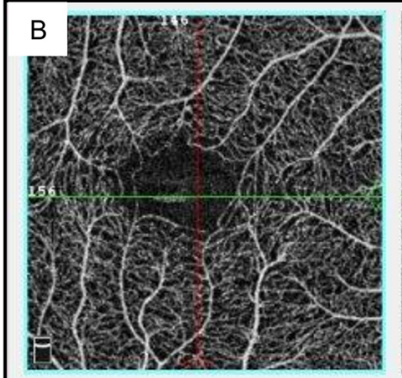

Mngio - Superficial

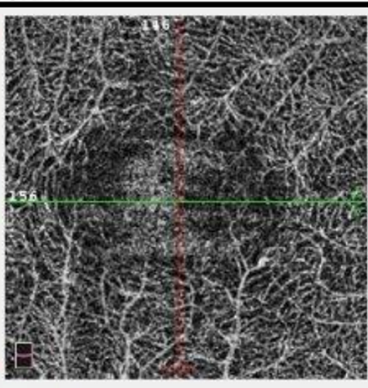

Angio-Deep

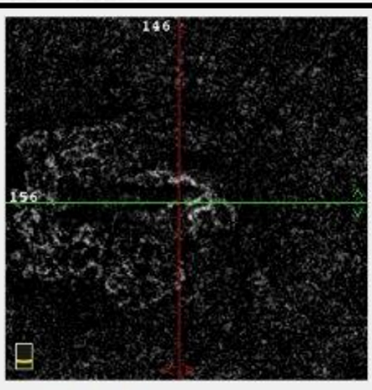

Angio - Outer Retina

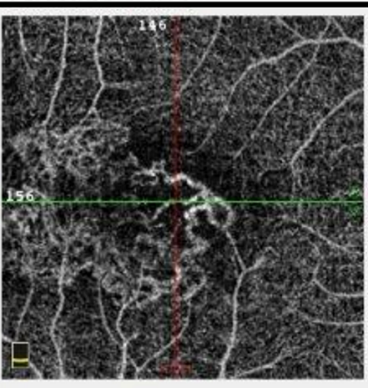

Angio-Choroid Capillary

Fig. 2 OCTA imaging showing A the 'pruned tree' pattern and $\mathbf{B}$ the 'tangled' pattern of new vessels morphology in different patients at the choriocapillaris segmentation

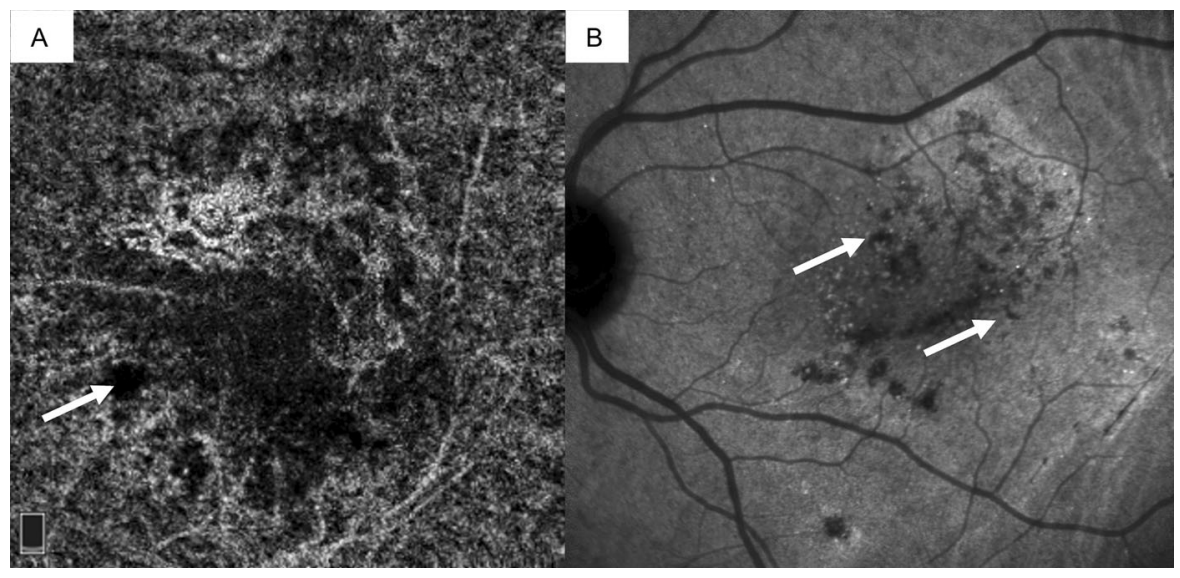

Fig. 3 ICGA imaging showing A 'dark areas' and B 'dark spots' (white arrows)

modify permeability and tissue perfusion. The clinical etiology of dark areas is a subject of debate: because these appear as a reduction in the diffuse reflectivity of the choriocapillaris, some authors consider this phenomenon to be due to a shadow effect of the neuroepithelium detachment, while for others it is the result of focal choriocapillary atrophy caused by a flow void due to large vessel compression (see also Pichi et al. [16]). In our opinion, the dark areas could represent a rarefaction of the choriocapillaris with reduced perfusion. About $80 \%$ of the patients in our study presented with this morphology, associated with subretinal fluid [27]. Conversely, the dark spots are clearly delimited lesions corresponding to hyporeflective dots on the ICGA. We considered these lesions as focal points of choriocapillary hypoperfusion, according to the degenerative features of the pathology.

Our study has three main limitations. First, the cohort of patients is too small to provide statistically significant data; second, there is a possible selection 
bias given that we selected patients receiving our second-level medical retina service; therefore, some differences in the time from clinical presentation to diagnosis could be present; third, all patients were naïve and the neovascular patterns could present different stages of evolution.

In conclusion, today, multimodal imaging represents a comprehensive approach that improves our understanding of CSC with FIPEDs. This study characterized the phenotype of hidden neovascular lesions in shape and morphology with OCTA analysis. The early and accurate detection of CNV is crucial for a good visual outcome, as anti-VEGF therapy represents an effective treatment for CNV in AMD [29], when diagnosed early. The new clinical features we described pose an interesting challenge for new therapeutical approaches in chronic CSC.

Authors' contribution Claudio Azzolini and Maurizio Chiaravalli supervised the study; Jennifer Cattaneo collected data; Laura Premoli and Cristian Metrangolo supervised the manuscript editing and statistics; Simone Donati and Jennifer Cattaneo performed the study and prepared the manuscript.

Funding Open access funding provided by Università degli Studi dell'Insubria within the CRUI-CARE Agreement. The study was conducted with research funds from Department of Ophthalmology, University of Insubria, Varese, Italy.

Data availability All data will be available by request.

\section{Declarations}

Conflicts of interest The authors declare no conflict of interest on the matter of the manuscript.

Ethical approval IRB approval was obtained considering a non-interventional observational study.

Consent to participate Patients provided written informed consent for data management and publication.

Informed consent Informed consent was obtained from all individual participants included in the study.

Open Access This article is licensed under a Creative Commons Attribution 4.0 International License, which permits use, sharing, adaptation, distribution and reproduction in any medium or format, as long as you give appropriate credit to the original author(s) and the source, provide a link to the Creative Commons licence, and indicate if changes were made. The images or other third party material in this article are included in the article's Creative Commons licence, unless indicated otherwise in a credit line to the material. If material is not included in the article's Creative Commons licence and your intended use is not permitted by statutory regulation or exceeds the permitted use, you will need to obtain permission directly from the copyright holder. To view a copy of this licence, visit http://creativecommons.org/licenses/by/4.0/.

\section{References}

1. Bennett G (1955) Central serous retinopathy. Br J Ophthalmol 39:605-618. https://doi.org/10.1136/bjo.39.10.605

2. Spaide RF, Campeas L, Haas A et al (1996) Central serous chorioretinopathy in younger and older adults. Ophthalmology 103:2070-2079. https://doi.org/10.1016/s01616420(96)30386-2

3. Eandi CM, Ober M, Iranmanesh R et al (2005) Acute central serous chorioretinopathy and fundus autofluorescence. Retina 25:989-993. https://doi.org/10.1097/00006982200512000-00006

4. Wang M, Munch IC, Hasler PW et al (2008) Central serous chorioretinopathy. Acta Ophthalmol 86:126-145. https:// doi.org/10.1111/j.1600-0420.2007.00889.x

5. Daruich A, Matet A, Dirani A et al (2015) Central serous chorioretinopathy: Recent findings and new physiopathology hypothesis. Prog Retin Eye Res 48:82-118. https://doi. org/10.1016/j.preteyeres.2015.05.003

6. Hage R, Mrejen S, Krivosic V et al (2015) Flat irregular retinal pigment epithelium detachments in chronic central serous chorioretinopathy and choroidal neovascularization. Am J Ophthalmol 159:890-903. https://doi.org/10.1016/j. ajo.2015.02.002

7. Mrejen S, Sarraf D, Mukkamala SK et al (2013) Multimodal imaging of pigment epithelial detachment: a guide to evaluation. Retina 33:1735-1762. https://doi.org/10.1097/IAE. 0b013e3182993f66

8. Spaide RF, Hall L, Haas, et al (1996) Indocyanine green video- angiography of older patients with central serous chorioretinopathy. Retina 16:203-213. https://doi.org/10. 1097/00006982-199616030-00004

9. Kim HC, Cho WB, Chung H (2012) Morphologic changes in acute central serous chorioretinopathy using spectral domain optical coherence tomography. Korean J Ophthalmol 26:347-354. https://doi.org/10.3341/kjo.2012.26.5.347

10. Chan SY, Wang Q, Wei WB et al (2016) Optical coherence tomographic angiography in central serous chorioretinopathy. Retina 36:2051-2058. https://doi.org/10.1097/IAE. 0000000000001064

11. Quaranta-El Maftouhi M, El Maftouhi A, Eandi CM (2015) Chronic central serous chorioretinopathy imaged by optical coeherence tomographic angiography. Am J Ophthalmol 160:581-587. https://doi.org/10.1016/j.ajo.2015.06.016

12. Dansingani KK, Balaratnasingam C, Klufas MA et al (2015) Optical coherence tomography angiography of shallow irregular pigment epithelial detachments in pachychoroid spectrum disease. Am J Ophthalmol 160:1243-1254.e2. https://doi.org/10.1016/j.ajo.2015.08.028

13. Fung AT, Yannuzzi LA, Freund KB (2012) Type 1 (subretinal pigment epithelial) neovascularization in central serous chorioretinopathy masquerading as neo-vascular 
age-related macular degeneration. Retina 32:1829-1837. https://doi.org/10.1097/IAE.0b013e3182680a66

14. Guo J, Tang W, Liu W, Chang Q, Xu G (2020) Clinical Features of flat irregular pigment epithelium detachment associated with choroidal neovascularization in chronic central serous chorioretinopathy. Retina. https://doi.org/10. 1097/IAE.0000000000002791

15. Gupta R, Chhablani J (2019) Flat irregular pigment epithelium detachment in central serous chorioretinopathy: correlation with choroidal neovascular membrane. Indian J Ophthalmol 67(8):1330-1332. https://doi.org/10.4103/ijo. IJO_2092_18

16. Pichi F, Morara M, Veronese C, Ciardella AP (2018) The overlapping spectrum of flat irregular pigment epithelial detachment investigated by optical coherence tomography angiography. Int Ophthalmol 38(3):975-983. https://doi. org/10.1007/s10792-017-0547-x

17. Fujimoto H, Gomi F, Wakabayashi, et al (2008) Y. Morphologic changes in acute central serous chorioretinopathy evaluated by Fourier-domain optical coherence tomography. Ophthalmology 115:1494-1500. https://doi.org/10. 1016/j.ophtha.2008.01.021

18. Van Velthoven MEJ, Verbraak FD, Garcia, et al (2005) Evaluation of central serous retinopathy with en face optical coherence tomography. Br J Ophthalmol 89:1483-1488. https://doi.org/10.1136/bjo.2005.073056

19. Bousquet E, Bonnin S, Mrejen S et al (2018) Optical coherence tomography angiography of flat irregular pigment epithelium detachment in chronic central serous chorioretinopathy. Retina 38:629-638. https://doi.org/10. 1097/IAE.0000000000001580

20. Liu R, Li J, Li Z et al (2016) Distinguish polypoidal choroidal vasculopathy from typical neovascular age-related macular degeneration based on Spectral Domain Optical Coherence Tomography. Retina 36:778-786. https://doi. org/10.1097/IAE.0000000000000794

21. Chung SE, Kang SW, Lee JH, Kim YT (2011) Choroidal thickness in polypoidal choroidal vasculopathy and exudative age-related macular degeneration. Ophthalmology 118(5):840-845. https://doi.org/10.1016/j.ophtha.2010.09. 012

22. Nicolò M, Rosa R, Musetti D et al (2017) Choroidal vascular flow area in central serous chorioretinopathy using swept-source optical coherence tomography angiography. Invest Ophthalmol Vis Sci 58(4):2002-2010. https://doi. org/10.1167/iovs.17-21417

23. Hwang H, Kim JY, Kim KT, Chae JB, Kim DY (2019) Flat irregular pigment epithelium detachment in central serous chorioretinopathy: a form of pachychoroid neovasculopathy? Retina. https://doi.org/10.1097/IAE.0000000000 002662

24. Miere A, Semoun O, Cohen SY et al (2015) Optical coherence tomography angiography features of subretinal fibrosis in age related macular degeneration. Retina 35:2275-2284. https://doi.org/10.1097/IAE.000000000000 0819

25. Liu T, Lin W, Zhou S, Meng X (2020) Optical coherence tomography angiography of flat irregular pigment epithelial detachments in central serous chorioretinopathy. $\mathrm{Br} \mathrm{J}$ Ophthalmol. https://doi.org/10.1136/bjophthalmol-2019315318

26. Souied EH, Miere A, Cohen SY et al (2016) Optical Coherence Tomography Angiography of Fibrosis in AgeRelated Macular Degeneration. Dev Ophthalmol 56:86-90. https://doi.org/10.1159/000442783

27. Karacorlu M, Sayman Muslubas I, Arf S, Hocaoglu M, Ersoz MG (2019) Membrane patterns in eyes with choroidal neovascularization on optical coherence tomography angiography. Eye (Lond) 33:1280-2128. https://doi.org/10. 1038/s41433-019-0415-1

28. Costanzo E, Cohen SY, Miere A et al (2015) (2015) Optical coherence tomography angiography in central serous chorioretinopathy. J Ophthalmol. https://doi.org/10.1155/ 2015/134783

29. Pece A, Azzolini C, Parodi MB et al (2012) Consensus on the diagnosis, treatment and follow-up of patients with agerelated macular degeneration eligible for ranibizumab. Expert Rev Ophthalmol 7(3):219-225. https://doi.org/10. 1586/eop. 12.24

Publisher's Note Springer Nature remains neutral with regard to jurisdictional claims in published maps and institutional affiliations. 